$\xi \ldots$

\title{
Fault Location on Series Compensated Power Transmission Lines Using Transient Spectrum
}

\author{
C.R. Jayamurthi ${ }^{1 *}$, A. Sowmiya ${ }^{2}$ \\ ${ }^{1}$ PG Scholar, Department Of Electrical And Electronics Engineering, Prist University, Thanjavur, Tamilnadu, India. \\ ${ }^{2}$ Assistant Professor, Department Of Electrical And Electronics Engineering, Prist University, Thanjavur, Tamilnadu, India. \\ *Corresponding Author E-Mail:Cjayamurthi555@Gmail.Com
}

\begin{abstract}
A Transmission line fault locality analyzing process for multi-circuit power transmission series compensated line up with phasor records as of smart electronic devices available at both split ends. During the case of unavailability of synchronize records, logical synchronization is acquire by means of an operative. During the power transmission process, the techniques consider untransposed line segment ensuing on top of two side of the power fault with the dispersed environment of transmission line. In favor of in the pink phase, current on two sides flow at the fault position be identical unlike exceptional current on two sides in a fault phase. This be checkered in excess of the line length along with the fault location is identify. The technique does not need the model of the series recompense located by at all locality on the line along with be capable of be use for both internal and external-circuit fault. It as well reflects on reactive element of the fault impedance for accurate fault position. The technique is analyzed with PSCAD/EMTDC simulation used for a multicircuit series-compensated line in Indian smart power grid. Estimation revise confirm the strength of the technique used for different faults.
\end{abstract}

Keywords: Fault Identification, series compensated power transmission line, reactive fault impedance, unsynchronized measurements, and untransposed line section.

\section{Introduction}

An electrical energy generation and distribution at remote location is delivering with transmission lines. Any faults occur or linked through the transmission lines, must live detect and isolated quickly to preserve a dependable power scheme and to suit everyday consumer requirements. A accepted of transmission system are sheltered with impedance relay. Even though impedance relay be use in nearly all security scheme, their performance be a smaller amount acceptable during series content transmission system. Impedance relay be designed through the supposition to facilitate the transmission line are inductive. Inclusions of capacitors in sequence through transmission lines make part of the transmission line capacitive bank, depending on the locality of the fault. This might direct to voltage source inversion, present source inversion, or together of current and voltage inversions. Remote Distance relay use to defend series compensated power transmission lines might mis-operate under this condition. Through faults, non-liner route of sequence capacitor bank and additional related mechanism (such as air-gap, gas filled spark and others) might as well shortest to sub-harmonic level or exponential dc counterbalance condition. This condition might occasionally direct to under-reach or damage problem.

A Distribution and Transmission lines are showing to fault to facilitate are cause by dissimilar reason such as short circuits, birds, and storms. Most of these fault consequence in routine damage of power lines which should be repair before persistent the column to repair. Transmission power line fault should be positioned truthfully toward permit maintenance crew toward appear on the landscape and refurbish the fault segment at the same time as soon as probable. Strong landscape and environmental layout origin several sections of power transmission lines toward are not easy to accomplish. Hence, robustness of the correct faults location purpose underneath a diversity of power system operating constraint and fault condition is a significant requirement. Generally, fast and accurate fault location will accelerate supply restoration and enhance the supply quality and reliability. In addition, this will diminish the customer problem. Therefore, fault location can be deliberate as one of the initial function to be real integrated into their transmission substation control system. Variety of fault-location algorithms include and to be developed and presented in the literature. The mainstream of them be base on an impedance principle, creation utilize of the original frequency voltages source and currents. A transmission line Fault-location algorithms base on traveling-wave occurrence, high frequency components of voltages and currents generate by fault, and false cleverness such the same as fuzzy neural network include in addition been urbanized.

\section{Related Works}

In [1] Ning Kang and Yuan Liao et al present an innovative approach for locating short-circuits fault on a double-circuit smart power transmission lines. In Transmission line various algorithms have been proposed formerly with the purpose of frequently need measurements record from one or two buses of the fault line. Though, such measurements could not forever be presented obtainable in several scenarios, depiction inapplicability of accessible method. To balance present method, this paper propose a novel, universal fault-location technique through harness transmission line voltage measurements at one or more buses, 
which cannot be there taken from the smart grid faulted line. The transmission line bus impedance matrix of every sequence network through the adding of a pretended bus next to the fault point is able to be resulting as a function of the transmission fault location. The transmission line fault location is able to in that case be there obtain based on the smart grid bus impedance matrix and voltage measurements. The distributed parameter line reproduction is utilized.

In [2] A. Gopalakrishnan, M. Kezunovic, S. M. McKenna, and D. M. Hamai et al presents previous work at Texas A\&M University lead toward the expansion of smart grid transmission line various fault location algorithms to facilitate be based on matched sample of the current and voltage data from the two ends of the feeder line. The column model worn during the algorithms is based on lump stricture model used for electrically small lines, or lossless dispersed parameter model in support of electrically lengthy lines. In this paper, the lossless line models are customized to account used for the series losses during the line. The line model equations be subsequently solving during the time domain to correctly locate the fault. Testing of the adapted algorithm is perform on a smart grid power system belong to the Western Area Power Administration. Extensive EMTP based simulations be used to produce data to facilitate be supplied as inputs to the different fault location algorithm.

In [3] Yuan Liao and Ning Kang et al present Novel smart grid power system transmission- line fault-location algorithms without require transmission line parameters. The current and voltage from both ends of a line to be in use as inputs and no synchronization is essential. Together Perrault and fault data might be utilized. The proposed method be based on a distributed constraint line model and, consequently, effusive regard as the impact of shunt capacitance of the transmission power line. Positive-sequence line parameters could as well be present estimated as a byproduct. No hypothesis on the source impedance and fault resistance is made. Estimation study based on Electromagnetic Transients Program (EMTP) simulation data express to facilitate the new method are capable to achieve Speedy and accurate location of fault into a transmission system be able to assist preservation engineers speedily pinpoint the fault component and achieve repair work along with, consequently, significantly reduce outage time.

In [4] Christos A. Apostolopoulos and George N. Korres et al presents A novel algorithm used for locate faults on power grid transmission lines without require line parameters. The algorithms utilize unsynchronized measurement of voltages and currents from both ends to be monitored of a transmission line with is formulate within conditions of the essential frequency phasor of the measured signals. Both Perrault with post fault phasor are process for influential they required distance toward fault and the synchronization phase. The calculation is performing in the beginning in favor of a lump parameter line models through neglect shunt capacitance effect. After that, these results are use as initial value used for an iterative process, where the impact of the shove capacitance of the line is considered. The planned 2-stage fault-location algorithm is suitable used for transposed along with untransposed transmission lines with be independent of the fault resistance and power source impedances.

In [5] Christos A. Apostolopoulos and George N. Korres et al presents A novel algorithm in favor of locating faults on doublecircuit power grid transmission lines with two-end unsynchronized current measurement.

The algorithm do not need line parameters, which is a essential step forward compare toward existing approach, which involve this information, consequently it can be measured as a settingsfree algorithms. Simply the positive-sequence current phasor throughout the fault be process in support of formative the required distance to fault and the synchronization phase angle, restraining as a result the amount of data required to be there transfer beginning every line terminal.

The proposed algorithm is derivative through apply the Kirchhoff's voltage law in the order of the parallel circuits' loops throughout the fault.

\section{Proposed System}

A transmission line fault analysis in both internal and external feeder with the help of series compensated dynamic algorithm. A technique in support of location of both internal and external circuit fault on dynamic algorithms analyzed. Series compensation methods which consider each fault segment as untransposed and the arc is model through both resistance value and inductance types. The fault location of the recompense is able to be at one end or some other location. A transmission line feeder monitoring using dynamic algorithm to measure the both end of transmission line with unsynchronized. A feeder synchronization operator is calculating using both end data on the technique to create a regular time orientation for the feeder ends. The equal opportunity restriction of the healthy phase currents in occurs fault line, at the accurate fault point, is subjugated to raise an accurate fault location indicator (which is zero at the correct fault location). Dynamic algorithm suitable solutions for fault location is determined by calculate this index over the transmission line length. The dynamic algorithm is exactly detected for accurate fault location.

\section{Series Capacitor's Overvoltage Protection System}

Series capacitor beside through its over-voltage safety protection system is frequently referred to as series compensating unit. In the event of any fault on transmission lines, the fault current flows through the series capacitors; as a result, the series capacitors are subjected to high stresses during the fault conditions. Therefore, defense of the capacitor from over voltages is of overriding importance. The overvoltage protection system of the series capacitor term as series capacitor protection unit (SCPU) is shown in Figure SCPU contains the series capacitor and other components which make available the primary and endorsement protection to the series capacitor. Different components of SCPU are discussed below in detail. The purpose of the MOV is to boundary the voltage crosswise the capacitor. At the same time it also directs a large amount of fault current through it. MOVs are designed in a way so that they can carry the fault modern for a maximum of 4-5 cycles. They are faster in action as they are always connected in parallel with series capacitor and require not being trigger by a control signal. Whenever there is a fault, MOV is ready for protection. Figure shows a fault scenario when MOV conducts. It can be observed that MOV conducts for a part of the cycle and for the rest of the part, the capacitor conducts.

\section{Transmission Line}

An overhead power transmission line is one of the major components in each feeder electric power system. A power Transmission lines unite the generating the power stations with load centers. As the power generate stations are far away from the load centers they run over hundreds of kilometers. Ever since fault is able to undermine the power system they are required to be isolated instantly. Fault analysis is especially momentous focus in power system manufacturing during classify that to clear fault quickly with restore power supply as soon as achievable with least amount disruption.

While a fault occurs on an electrical power transmission line, it is especially significant to identify it and to uncover its location during arrange to create essential repairs and to reinstate power as almost immediately as feasible. The time required to decide the fault point alongside the line will affect the quality of the power delivery. For that reason, a precise fault location on the transmission line is a significant requirement in support of a permanent fault. 


\section{Faults in Transmission Lines}

To increase the population an electric power from generate stations to remote load centers, transmission feeder lines are used. Due to lightening, mismatched operation, power overload problem, short circuits problem on transmission line, human errors, faulty equipment problem and aging, faults might arise on these transmission lines. When fault occur on transmission line, the faulted phase voltage decreases and huge currents resolve flow which can burn out the mechanism if not interrupted quickly. In feeder transmission lines with a three-phase power source, there are 10 types of faults to facilitate be able to occur. Throughout the fault the impedance, Zfag, is not essentially zero (bolted) except it strength contain a non-zero impedance although at rest much lesser than the line impedance.

\section{Block diagram}
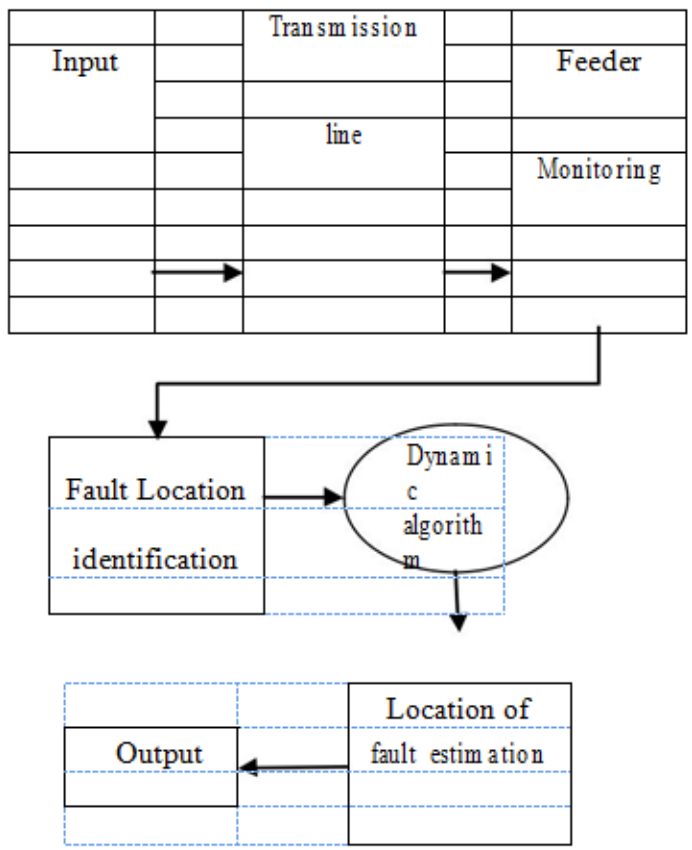

Fig 1: Block diagram

\section{Fault Location}

The above figure the electric power is transmit to transmission line on customer side is occurs short circuit or overload faults and some other faults. A Dynamic algorithm is easily identifying the fault and product the distribution system can really reduced the time to recovery. The transmission line voltage and current continuously monitoring on feeder line. The feeder voltage is dropping or infinity or short circuit means to identity and product the substation with the help of series compensated dynamic algorithm. The transmission line power quality also measured and secured power transmission. Due to the fast development of large scale smart grid is securely products on series compensated transmission dynamic algorithms. Dynamic algorithm is intelligent methods to work with fault identification and their good performance.

\section{Adaptive Transmission Fault Location}

The major aspire of adaptive transmission line fault location algorithms is to achieve an improved fault location accuracy. The idea of adaptive algorithm fault location on transmission lines boil along to appropriate evaluation of line parameters and system transmission line impedance. The ecological conditions and operation record of the transmission line affect its sag. As the performer total line current increases, its temperature increases and as a result its sag. The line resistance change among the line temperature. In addition, the line reactance will change since it depends on the distance between the phase conductors which is pretentious by the line sag. Therefore, the indecision of the line parameters could affect substantially the accuracy of the fault location. The effect of such uncertainty can reach up to 6-7\% if the parameters vary $20 \%$ of the practical parameters. It is worth mentioning that the power utility usually provides very ideal parameters of the line that do not take into consideration its operation history. With normal operation, PMU can measure the voltage at both ends of the line along with its current. The line parameters can be calculated using the prefault phase and amplitude capacity of the voltages and currents.

\section{Proposed Fault Location Method}

A Transmission line fault location analyzed method for multi circuit series compensated transmission line. The quality of fault analysis in over the transmission line to identify the correct location with dynamic adaptive algorithm. The fault analysis on feeder line the relation between phases voltages present at the line with unbalanced voltage analysis on transmission line. The unbalanced voltage is detected and product tom the substation and also exact fault location identified. The fault occur in transmission line both resistance and inductance are calculated.

\section{Fault Location in Power System Networks}

Distribution networks fault location detection and identified creates new problems compare through the similar task in HV and EHV transmission lines. In fault locator identified on HV and EHV networks. In such a container, the Dynamic fault locator algorithm is analyzed voltage and current into a single number living being a distance to fault. The distribution and transmission line dynamic fault locator to exactly measure the voltage and current and identify the fault location.

\section{Output Result}

\section{Overall Process}

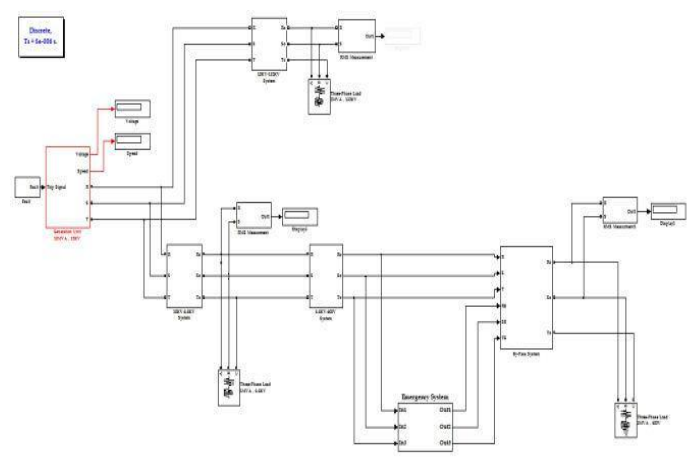

\section{Generation Unit}

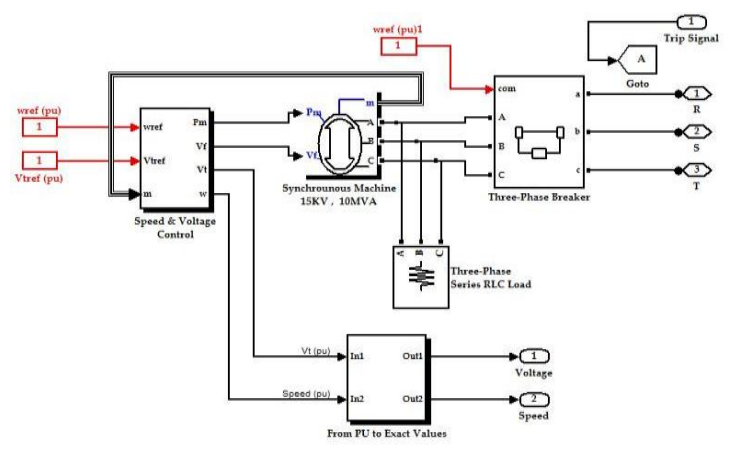




\section{Voltage and Current Output}

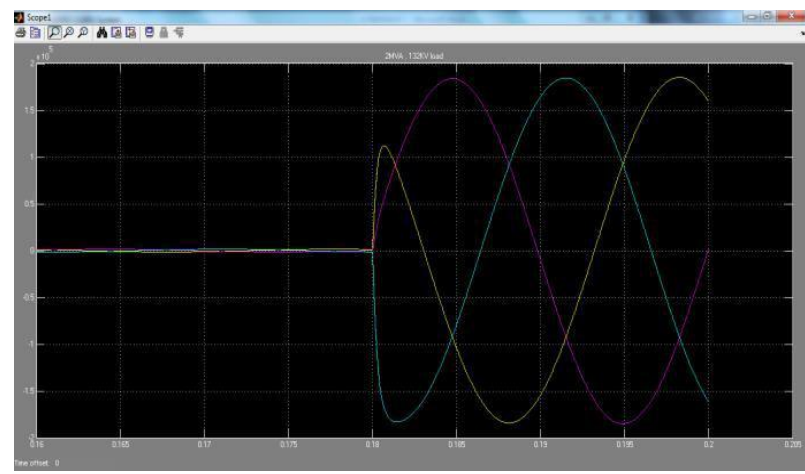

\section{PWM Generator Output}

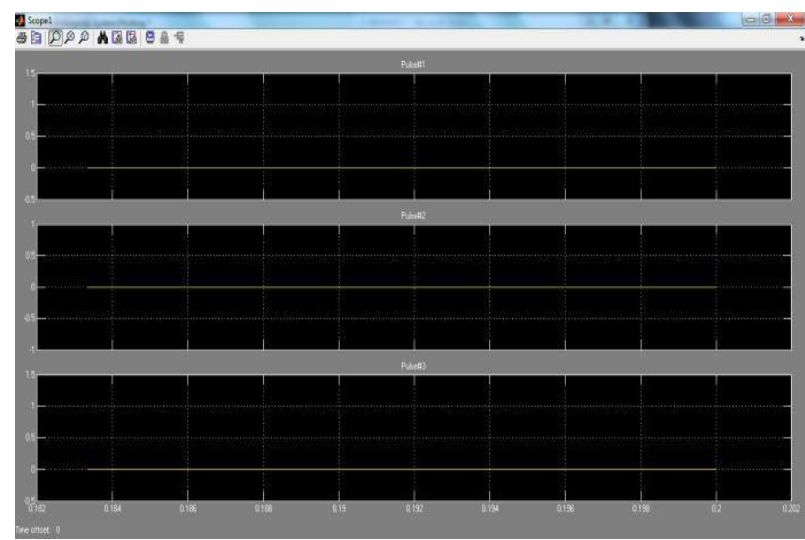

\section{Inverter Output}

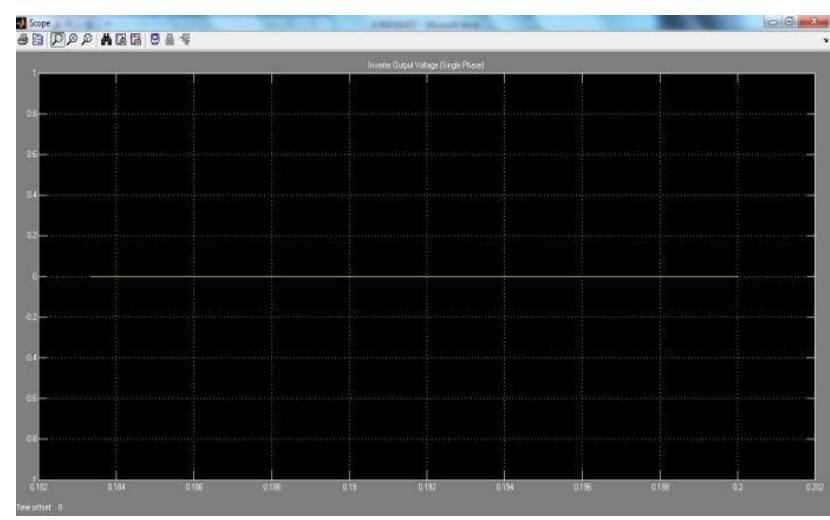

\section{Bypass System Output}

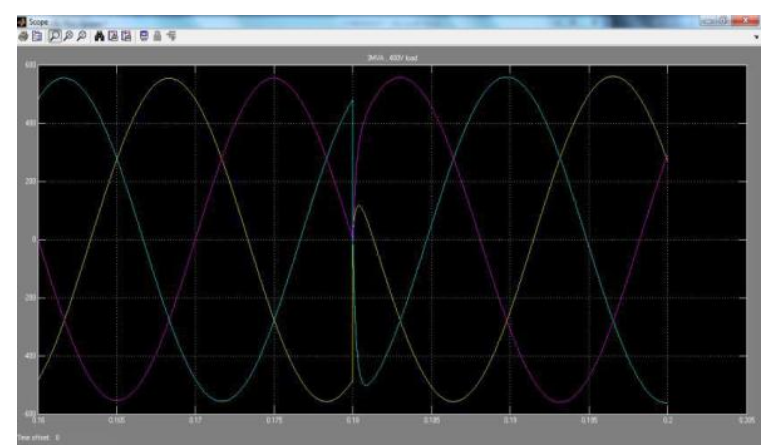

\section{Conclusion}

A transmission line fault location identification for multi-series compensated line with data analyzed in both ends. This method efficiently analyzed and easily applied to series compensation transmission line. The fault occurs in transmission line the current and voltage value are infinity. The transmission line resistance and inductance values are calculated the dynamic fault locator algorithm. The proposed dynamic algorithm to monitor the transmission line on both sides and fault is detected. The fault detection on transmission line with detection and identified multiseries compensation transmission line using fault locator. These techniques used on internal and external substation. The line parameters are analyzed on transmission line. The fault location detection is very accurately detected and intimated in this system overall system performance is good.

\section{References}

[1] Kezunovic M, "Smart fault location for smart grids", IEEE Trans. Smart Grid, Vol.2, No.1, (2011), pp. 11-22.

[2] Saha MM, Izykowski JJ \& Rosolowski E, "Fault Location on Power Networks, ser. Power Systems", Springer-Verlag London Ltd, (2010).

[3] Kawady T \&Stenzel J, "A practical fault location approach for double circuit transmission lines using single end data", IEEE Trans. Power Del., Vol.18, No.4, (2003), pp.1166-1173.

[4] Izykowski J, Rosolowski E \& Saha MM, "Locating faults in parallel transmission lines under availability of complete measurements at one end", IEE Proceedings- Generation, Transmission and Distribution, Vol.151, No.2, (2004), pp.268-273.

[5] Song G, Suonan J \& Ge Y, "An accurate fault location algorithm for parallel transmission lines using one-terminal data", International Journal of Electrical Power \& Energy Systems, Vol.31, No.2, (2009), pp.124- 129.

[6] Takagi T, Yamakoshi Y, Yamaura M, Kondow R \& Matsushima T, "Development of a new type fault locator using the one-terminal voltage and current data", IEEE Trans. PAS, Vol. PAS-101, No.8, (1982), pp.2892-2898

[7] Kang SH, Ahn YJ, Kang YC \& Nam SR, "A fault location algorithm based on circuit analysis for untransposed parallel transmission lines", IEEE Trans. Power Del., Vol.24, No.4. (2009), pp.1850-1856.

[8] Izykowski J, Molag R, Rosolowski E \& Saha MM, "Accurate location of faults on power transmission lines with use of two-end unsynchronized measurements", IEEE Trans. Power Del., Vol.21, No.2, (2006), pp.627-633.

[9] Izykowski J, Rosolowski E, Balcerek P, Fulczyk M \& Saha MM, "Accurate noniterative fault-location algorithm utilizing two-end unsynchronized measurements", IEEE Trans. Power Del., Vol.26, No.2, (2011), pp.547-555.

[10] Brahma SM, "Fault location scheme for a multi-terminal transmission line using synchronized voltage measurements", IEEE Transactions on Power Delivery, Vol.20, No.2, (2005), pp.1325-1331.

[11] Brahma SM \& Girgis AA, Fault location on a transmission line using synchronized voltage measurements", IEEE Trans. Power Del., Vol.19, No.4, (2004), pp.1619-1622.

[12] Yu CS, "An unsynchronized measurements correction method for two terminal fault-location problems", IEEE Trans. Power Del., Vol.25, No.3, (2010), pp.1325-1333.

[13] Ma J., Shi Y., Ma W. \& Wang Z, "Location method for inter-line and grounded faults of double-circuit transmission lines based on distributed parameters", IEEE Trans. Power Del.,(2014).

[14] Kang N \& Liao Y, "Double-circuit transmission-line fault location with the availability of limited voltage measurements", IEEE Trans. Power Del., Vol.27, No.1, (2012), pp.325-336.

[15] Gopalakrishnan A, Kezunovic M, McKenna SM \& Hamai DM, "Fault location using the distributed parameter transmission line model", IEEE Trans. Power Del., Vol.15, No.4, (2000), pp.1169-1174. 